\title{
Approaches to Solve MID_CPLP Problem: Theoretical Framework and Empirical Investigation
}

\author{
Renduchintala Raghavendra Kumar Sharma1, Pritee Agarwal' \\ ${ }^{1}$ Department of Industrial and Management Engineering, Indian Institute of Technology, Kanpur, India \\ ${ }^{2}$ Department of Mathematics and Statistics, Indian Institute of Technology, Kanpur, India \\ Email: rrks@iitk.ac.in, pritee.iitk@gmail.com, pritee@iitk.ac.in
}

Received 14 March 2014; revised 14 April 2014; accepted 21 April 2014

Copyright (C) 2014 by authors and Scientific Research Publishing Inc.

This work is licensed under the Creative Commons Attribution International License (CC BY). http://creativecommons.org/licenses/by/4.0/

(c) () Open Access

\begin{abstract}
In two-stage warehouse location problem, goods are moved from plants to warehouses at stage-1 (which are larger sized warehouses), and from there to warehouses at stage-2 (which are smaller sized warehouses); and finally to the markets. We aim to minimize the sum of location costs of the warehouses at stage-1 and stage-2; plus the total distribution cost of goods to the markets. In this paper two-stage capacitated warehouse location problem (TSCWLP) is vertically decomposed into the smaller problems, which is attained by relaxing the associated flow balance constraints. This leads to three different versions of Capacitated Plant Location Problem (CPLP) referred as RHS_CPLP, MID_CPLP and LHS_CPLP (Verma and Sharma [1]). In this paper MID_CPLP is reduced to RHS_CPLP and a single constraint 0-1 Knapsack problem by relaxing a difficult constraint. Interesting results and conjectures are given. Later two more valid constraints are added to MID_CPLP which are relaxed further to get additional results.
\end{abstract}

\section{Keywords}

TSCWLP, Location, Transportation, Distribution

\section{Introduction and Literature Survey}

In country like large geographical area, such as India, distribution of goods such as fertilizer, cement, edible oil, sugar, food grains etc, is close in stages. From large sized plants, goods are moved to larger sized warehouses, from larger sized warehouses to smaller sized warehouses and finally to markets/customers. It is to be decided that where to locate warehouses (among potential locations for large sized warehouses as well as smaller sized 
warehouses). It is required to minimize sum total of cost of location of warehouse and cost of distribution of goods. Stage wise location-distribution has been attempted by Geoffrion and Graves [2], Sharma [3], Sharma and Berry [4] and Verma and Sharma [5]. In this paper we attempt a two-stage warehouse location problem and alongside give a novel method to solve MID_CPLP that results due to vertical decomposition developed for solution. Facility location problems form an important class of integer programming problems, with application in the distribution and transportation industries. In any organization, one of the most important strategic decisions is locating facilities viz. factories, plants or warehouses and their suitable stages. While locating a facility, the most prioritized criterion is a good service level. However, achievement of an economic optimality is also a key decisive factor. Based on the number of stages between the producing facility and the market, there are different types of facility location problems like plant location problem, single-stage/two-stage warehouse location problem. A review of the literature on location problems can be found in (Sahin and Sural [6], ReVelle and Eiselt [7], ReVelle, Eiselt and Daskin [8] and Brandeau and Chiu [9]). Multistage warehouse location problems are frequently occurring in real life; see (Geoffrion and Graves [2], Sharma [3] and Sharma [10]). TSCWLP problem has been studied by Verma and Sharma [1]. In Section 2, we give formulation of TSCWLP, few relaxations of MID_CPLP; and also give a few results. In the Section 3 we give empirical investigation and related statistical significance. Finally in Section 4 we conclude.

\section{Formulation and Relaxations of TSCWLP}

\subsection{Problem Formulation of TSCWLP}

In this section, we propose the formulation of TSCWLP using the style of Sharma [3], Sharma and Namdeo [11], Sharma and Sharma [12]. Verma and Sharma [5] developed a variety of constraints that link real and 0-1 integer variables. They have also developed some strong constraints based on Sharma and Berry [4].

\subsubsection{Index Used}

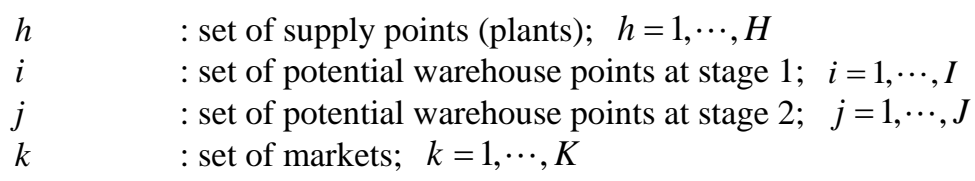

\subsubsection{Definition of Constants}

$D_{k} \quad$ : Demand for the commodity at market " $k$ "

$d_{k} \quad: D_{k} / \sum D_{k}$, Demand at market " $k$ " as a fraction of total market demand

$S_{h} \quad$ : Supply available at plant " $h$ "

$s_{j} \quad: \quad S_{h} / \sum D_{k}$, Supply available at plant ' $h$ ' as a fraction of the total market demand

fws $1_{i} \quad$ : Fixed cost of locating a warehouse at " $i$ "

$f w s 2_{j} \quad$ : Fixed cost of locating a warehouse at " $j$ "

$C A P W S 1_{i}$ : Capacity of a stage 1 warehouse " $i$ "

CAPWS2 $2_{j}$ : Capacity of a stage 2 warehouse " $j$ ”

capws $1_{i} \quad: C A P W S 1_{i} / \sum D_{k}$, Capacity of whs- 1 at " $i$ "'as a fraction of the total market demand

capws $2_{j} \quad: \quad C A P W S 2_{i} / \sum D_{k}$ Capacity of whs- 2 at " $j$ " as a fraction of the total market demand

${ }_{c p w s} 1_{h i} \quad$ : Cost of transporting $\sum D_{k}$ goods from plant " $h$ " to whs-1 "i”"

cws $1 w s 2_{i j}$ : Cost of transporting $\sum D_{k}$ goods from whs-1 " $i$ " to whs2 " $j$ "

$c w s 2 m_{j k} \quad$ : Cost of transporting $\sum D_{k}$ goods from whs-2 “j” to market " $k$ "

\subsubsection{Definition of Variables}

$X P W S 1_{h i} \quad$ : Quantity of commodity transported from plant " $h$ ” to whs-1 “i”

${ }_{x p w s} 1_{h i} \quad: X P W S 1_{h i} / \sum D_{k}$, Quantity transported from " $h$ " to " $i$ " as fraction of total demand

$X W S 1 W S 2_{i j}$ : Quantity of commodity transported from whs-1 " $i$ " to whs-2 " $j$ ”

$X w s 1 w s 2_{i j} \quad: X W S 1 W S 2_{i j} / \sum D_{k}$, Quantity transported from “i” to " $j$ ” as fraction of total demand

$X W S 2 M_{j k} \quad$ : Quantity of commodity transported from whs-2 “j” to market " $k$ "

$X w s 2 m_{j k} \quad: X W S 2 M_{j k} / \sum D_{k}$, Quantity transported from “ $j$ ” to " $k$ " as fraction of total demand

$y w s 1_{i} \quad: 1$ if stage 1 warehouse is located at " $i$ ", 0 otherwise 
$y w s 2_{j} \quad: 1$ if stage 2 warehouse is located at “ $j$ ”, 0 otherwise

\subsubsection{Mathematical Formulation}

The cost minimization problem for the TSCWLP can be written as mixed 0-1 integer linear programming problem as given in the formulation below.

\section{Objective Function:}

\section{Minimize}

$$
\begin{aligned}
Z= & \sum_{h, i} x p w s 1_{h i} * c p w s 1_{h i}+\sum_{i, j} x w s 1 w s 2_{i j} * c w s 1 w s 2_{i j}+\sum_{j, k} x w s 2 m_{h i} * c w s 2 m_{j k} \\
& +\sum_{i} f w s 1_{i} * y w s 1_{i}+\sum_{j} f w s 2_{j} * y w s 2_{j}
\end{aligned}
$$

This can be written as:

Minimize $Z=f_{1}+f_{2}+f_{3}$, where

$$
\begin{aligned}
& f_{1}\left(x p w s 1_{h i}, c p w s 1_{h i}, y w s 1_{i}, f w s 1_{i}\right)=\min _{x p w s 1, y w s 1} \sum_{h, i} x p w s 1_{h i} * c p w s 1_{h i}+\frac{1}{2} \sum_{i} y w s 1_{i} * f w s 1_{i} \\
& f_{2}\left(x w s 1 w s 2_{i j}, c w s 1 w s 2_{i j}, y w s 1_{i}, y w s 2_{j}, f w s 1_{i}, f w s 2_{j}\right) \\
& =\min _{x w s 1 w s 2, y w s 1, y w s 2} \sum_{i, j} x w s 1 w s 2_{i j} * c w s 1 w s 2_{i j}+\frac{1}{2} \sum_{i} y w s 1_{i} * f w s 1_{i}+\frac{1}{2} \sum_{j} y w s 2_{j} * f w s 2_{j} \\
& f_{3}\left(x w s 2 m_{j k}, c w s 2 m_{j k}, y w s 2_{j}, f w s 2_{j}\right)=\min _{x w s 2 m, y w s 2} \sum_{j, k} x w s 2 m_{j k} * c w s 2 m_{j k}+\frac{1}{2} \sum_{j} y w s 2_{j} * f w s 2_{j}
\end{aligned}
$$

Subject to:

$$
\begin{gathered}
\sum_{h, i} x p w s 1_{h i}=1 \\
\sum_{i, j} x w s 1 w s 2_{i j}=1 \\
\sum_{j, k} x w s 2 m_{j k}=1 \\
x p w s 1_{h i} \leq y w s 1_{i} s_{h}, \quad \forall h, i, \\
x w s 2 m_{j k} \leq y w s 2_{j} d_{k}, \quad \forall j, k, \\
x p w s 1_{h i} \leq y w s 1_{i}, \forall h, i, \\
x w s 2 m_{j k} \leq y w s 2_{j}, \forall j, k, \\
\sum_{h} x p w s 1_{h i} \leq y w s 1_{i} c a p w s 1_{i}, \quad \forall i, \\
\sum_{j} x w s 1 w s 2_{i j} \leq y w s 1_{i} c a p w s 1_{i}, \quad \forall i, \\
\sum_{i} x w s 1 w s 2_{i j} \leq y w s 2_{j} c a p w s 2_{j}, \quad \forall j, \\
\sum_{k} x w s 2 m_{j k} \leq y w s 2_{j} c a p w s 2_{j}, \quad \forall j, \\
\sum_{i} x p w s 1_{h i} \leq s_{h}, \quad \forall h, \\
\sum_{j} x w s 1 w s 2_{i j} \leq c a p w s 1_{i}, \forall i, \\
\sum_{i} x w s 1 w s 2_{i j} \leq c a p w s 2_{j}, \forall j,
\end{gathered}
$$




$$
\begin{gathered}
\sum_{j} x w s 2 m_{j k}=d_{k}, \forall k, \\
x p w s 1_{h i} \geq 0, \forall h, i, \\
x w s 1 w s 2_{i j} \geq 0, \forall i, j, \\
x w s 2 m_{j k} \geq 0, \forall j, k, \\
y w s 1_{i}=(0,1), \forall i, \\
y w s 2_{j}=(0,1), \forall j, \\
\sum_{i} y w s 1_{i} c a p w s 1_{i} \geq 1 \\
\sum_{j} y w s 2_{j} c a p w s 2_{j} \geq 1 \\
\sum_{i} c a p w s 1_{i} \geq 1 \\
\sum_{j} c a p w s 2_{j} \geq 1 \\
\sum_{h} x p w s 1_{h i}=\sum_{k} x w s 1 w s 2_{i j}, \forall i, \\
\sum_{i} x w s 1 w s 2_{i j}=\sum_{k} x w s 2 m_{j k}, \quad \forall j,
\end{gathered}
$$

Since $\sum d_{k}=1$, constraints 2, 2(a) and 2(b) ensure that flow across stages is equal to total demand by all the markets. Constraints 3(a) and 3(b) are strong linking constraints (see Sharma and Berry [4] and Verma and Sharma [5]). Equations 4(a) and 4(b) are weak linking constraints. Equations 5, 5(a-i), 5(a-ii) and 5(b) strong capacity constraints (see Sharma and Berry [4]). Equation 6 ensures the flow from plant is less than supply available at that plant. Equations 6(a-i) and 6(a-ii) ensure that throughput form a warehouse is less than or equal to its capacity. Equation 6(b) ensures that the quantity received at a market is equal to its demand. Equations 7 , 7(a) and 7(b) are non-negativity restrictions on real variables. Equations 8(a) and 8(b) are 0-1 restrictions on binary location variables. Equations 9(a) and 9(b) ensure that located capacity is more than or equal to the total demand of the markets. Equations 10(a) and 10(b) ensure that total average capacity is more than market demand at each stage. Equations 11(a) and 11(b) are flow balance constraints (inflow is equal to outflow) at each of the warehouses. By relaxing different constraints, various relaxations can be obtained as Lagrangian relaxation (LR). LR is a relaxation technique, which works by moving hard constraints into the objective to impose a penalty on the objective if they are not satisfied. This is easier to solve than the original problem. An optimal objective value of the Lagrangian relaxed problem, for a given set of multipliers, provides a lower bound (in the case of minimization) for the optimal solution to the original problem. The best lower bound can be derived by updating the multipliers by a dual ascent procedure. An upper bound on the optimal solution of the original problem can be derived by using the information obtained from the LR to construct a feasible solution to the original problem. This is normally done by applying some heuristic. In the next section, we present vertical decomposition approach for solving TSCWLP and variety of LR and LP relaxations.

\subsection{Lagrangian Relaxation of TSCWLP}

Proceeding in a manner similar to Verma and Sharma [13], TSCWLP is also vertically decomposed into three sub problems viz. LHS_CPLP, MID_CPLP and RHS_CPLP. In this approach, stages of warehouses are vertically decomposed to get smaller sized problems, which are relatively easier to solve. 1st LR of this formulation is obtained by relaxing flow balance constraints (11(a)) and (11(b)) with suitable multipliers $\lambda 1$ and $\lambda 2$ respectively. In main problem, we note that constraint set (11(a)) connects the "xpws $1_{h i}$ " and " $x w s 1 w s 2_{i j}$ " variables. Similarly, constraint set (11(b)) connects the " $x w s 1 w s 2_{i j}$ " and " $x w s 2 m_{j k}$ " variables. If these two sets of constraints are relaxed, main problem will be separated into three sub-problems. One problem with "xpws $1_{h i}$ " and 
" $y w s 1_{i}$ ", called as LHS_CPLP, the other with " $x w s 1 w s 2_{i j}$ ", “ $y w s 1_{i}$ ” and " $y w s 2_{j}$ ” to be called as MID_CPLP, and the last problem with " $x w s 2 m_{j k}$ " and " $y w s 2_{j}$ " called as RHS_CPLP. Verma and Sharma [13] have already attempted RHS_CPLP and LHS_CPLP. Here different relaxations of MID_CPLP are developed and relationship is obtained for them.

\subsubsection{LHS_CPLP}

$$
\begin{aligned}
Z_{L_{L H S} C P L P} & =f_{1}\left(x p w s 1_{h i},\left(c p w s 1_{h i}+\lambda 1_{i}\right), y w s 1_{i}, f w s 1_{i}\right) \\
& =\min _{x p w s 1, y w s 1_{h, i}} \sum_{i} x p w s 1_{h i} *\left(c p w s 1_{h i}+\lambda 1_{i}\right)+\frac{1}{2} \sum_{i} y w s 1_{i} * f w s 1_{i}
\end{aligned}
$$

s.t.: (2), (3), (4), (5), (6), (7), (8(a)), (9(a)), (10(a)).

\subsubsection{MID_CPLP}

$$
\begin{aligned}
Z_{\text {MID_CPLP }_{-}} & =f_{2}\left(x w s 1 w s 2_{i j},\left(c w s 1 w s 2_{i j}-\lambda 1_{i}+\lambda 2_{j}\right), y w s 1_{i}, y w s 2_{j}, f w s 1_{i}, f w s 2_{j}\right) \\
& =\min _{x w s 1 w s 2, y w s 1, y w s 2} \sum_{i, j} x w s 1 w s 2_{i j} *\left(c w s 1 w s 2_{i j}-\lambda 1_{i}+\lambda 2_{j}\right)+\frac{1}{2} \sum_{i} y w s 1_{i} * f w s 1_{i}+\frac{1}{2} \sum_{j} y w s 2_{j} * f w s 2_{j}
\end{aligned}
$$

s.t.: (2(a)), (5(a-i)), (5(a-ii)), (6(a-i)), (6(a-ii)), (7(a)), (8(a)), (9(a)), (10(a)), (8(b)), (9(b)), (10(b)).

\subsubsection{RHS_CPLP}

$$
\begin{aligned}
Z_{\text {RHS }} C P L P & =f_{3}\left(x w s 2 m_{j k},\left(c w s 2 m_{j k}-\lambda 2_{j}\right), y w s 2_{j}, f w s 2_{j}\right) \\
& =\min _{x w s 2 m, y w s 2} \sum_{j, k} x w s 2 m_{j k} *\left(c w s 2 m_{j k}-\lambda 2_{j}\right)+\frac{1}{2} \sum_{j} y w s 2_{j} * f w s 2_{j}
\end{aligned}
$$

s.t.: (2(b)), (3(b)), (4(b)), (5(b)), (6(b)), (7(b)), (8(b)), (9(b)) and (10(b)).

\subsubsection{Original relaxed TSCWLP}

$$
Z_{\text {ori }}=\max _{\lambda 1_{i}, \lambda 2_{j}}\left(Z_{L_{\text {LS }} C P L P}+Z_{\text {MID_CPLP }_{-}}+Z_{\text {RHS }_{-} C P L P}\right)
$$

s.t.: (2)-(7), (2(a)), (5(a-i)),(5(a-ii)), (6(a-i)), (6(a-ii)), (7(a))-(10(a)), (2(b))-(10(b)).

We focus on MID_CPLP in this chapter.

\subsection{A Few Theoretical Results on MID_CPLP}

We relax constraint (5(a-ii)) by attaching multiplier $\lambda 3_{j}$ to get,

\subsubsection{MID_CPLP $\left(\lambda 3_{j}\right)$}

$$
\begin{aligned}
Z_{\text {MID_CPLP }}= & f_{2}\left(x w s 1 w s 2_{i j},\left(c w s 1 w s 2_{i j}-\lambda 1_{i}+\lambda 2_{j}+\lambda 3_{j}\right), y w s 1_{i}, y w s 2_{j}, f w s 1_{i}, f w s 2_{j}\right) \\
= & \min _{x w s 1 w s 2, y w s 1_{1}, y w s 2} \sum_{i, j} x w s 1 w s 2_{i j} *\left(c w s 1 w s 2_{i j}-\lambda 1_{i}+\lambda 2_{j}+\lambda 3_{j}\right) \\
& +\frac{1}{2} \sum_{i} y w s 1_{i} * f w s 1_{i}+\frac{1}{2} \sum_{j} y w s 2_{j} * f w s 2_{j}-\sum_{j} c a p w s 2_{j} * y w s 2_{j} * \lambda 3_{j}
\end{aligned}
$$

s.t.: (2(a)), (5(a-i)), (6(a-i)), (6(a-ii)), (7(a)), (8(a)), (9(a)), (10(a)), (8(b)), (9(b)), (10(b)).

$M I D_{-} C P L P\left(\lambda 3_{j}\right)$ problem will be separated into two sub-problems: $C P L P{ }_{-} R\left(\lambda 3_{j}\right)$ (Verma and Sharma [5]) and one 0-1 knapsack problem (single constraint).

1) CPLP_R $\left(23_{j}\right)$

$$
Z_{C P L P_{-} R}=\min _{x w s 1 w s 2, y w s 1, y w s 2} \sum_{i, j} x w s 1 w s 2_{i j} *\left(c w s 1 w s 2_{i j}-\lambda 1_{i}+\lambda 2_{j}+\lambda 3_{j}\right)+\frac{1}{2} \sum_{i} y w s 1_{i} * f w s 1_{i}
$$


s.t.: (2(a)), (5(a-i)), (6(a-i)), (6(a-ii)), (7(a)), (8(a)), (9(a)), (10(a)), (10(b)).

2) 0-1_KSP $\left(\lambda 3_{j}\right)$

$$
Z_{0-1 \_K S P}=\frac{1}{2} \sum_{j} y w s 2_{j} * f w s 2_{j}-\sum_{j} c a p w s 2_{j} * y w s 2_{j} * \lambda 3_{j}
$$

s.t.: (8(b)), (9(b)).

\subsubsection{Relaxation of CPLP_R $\left(\lambda 3_{j}\right)$}

Now CPLP $-R\left(\lambda 3_{j}\right)$ is attempted in literature by three different relaxations. They are R1, R2 and R3 respectively. When integrality restriction on $y w s 1_{i}$ is relaxed we obtain (12(a)).

$$
0 \leq y w s 1_{i} \leq 1, \forall i,
$$

\section{1) CPLP_R1 $\left(23_{j}\right)$}

(1(f)); Subject to: (2(a)), (5(a-i)), (6(a-i)), (6(a-ii)), (7(a)), (10(a)), (10(b)) and (12(a)).

Result 1: R1 is equal to strong LP relaxation of $C P L P \_R\left(\lambda 3_{j}\right)$ (Sharma and Berry [4], Verma and Sharma [5]). Here the restriction on yws $1_{\mathrm{i}}$ is relaxed by (12(a)).

Proof: It is easy to see.

\section{2) CPLP_R2 $\left(\lambda 3_{j}\right)$}

In this $\mathrm{LR}$ a constraint introducing upper $\left(\mathrm{P}_{\mathrm{U} 1}\right)$ and lower $\left(\mathrm{P}_{\mathrm{L} 1}\right)$ limits on the number of open plants is added up, Cornuejols, Sridharan and Thizy [14] i.e.

$$
P_{L 1 ?} \leq \sum_{i} y w s 1_{i} \leq P_{U}, \forall i,
$$

This LR was proposed first by Christofides and Beasley [15] and later used Verma and Sharma [5] among others and is obtained by dualizing (2(a)) and (6(a-ii)). Let $\lambda x w s 1 w s 2, \lambda x w s 1 w s 2_{j}$ be the Lagrangian multipliers associated with (2(a)) and (6(a-ii)) respectively.

$$
\begin{gathered}
Z_{R 2}=\min _{x w s 1 w s 2, y w s 1, y w s 2} \sum_{i, j}\left(c w s 1 w s 2_{i j}-\lambda 1_{i}+\lambda 2_{j}+\lambda 3_{j}+\lambda x w s 1 w s 2+\lambda x w s 1 w s 2_{j}\right) * x w s 1 w s 2_{i j} \\
+\frac{1}{2} \sum_{i} y w s 1_{i} * f w s 1_{i}-\lambda x w s 1 w s 2-\sum_{j} \lambda x w s 1 w s 2_{j} * c a p w s 2_{j}
\end{gathered}
$$

Subject to: (5(a-i)), (6(a-i)), (7(a)), (10(a)), (10(b)), (8(a)) and (13(a)).

3) CPLP_R3 ( $\left.23_{j}\right)$

(1(h)); Subject to: (5(a-i)), (6(a-i)), (7(a)), (8(a)), (9(a)), (10(a)) and (10(b)). It is also attempted by first by Nauss [16] and later by Verma and Sharma [5]. We have following result due to Verma and Sharma [5].

Result 2: $C P L P_{-} R 1\left(\lambda 3_{j}\right) \leq C P L P_{-} R 2\left(\lambda 3_{j}\right) \leq C P L P_{-} R 3\left(\lambda 3_{j}\right)$.

If we add the objective function value of the $0-1$ knapsack problem in the objective value of CPLP_R then we get the objective value of the $M I D \_C P L P \_R$. Hence, the same relaxation along with their relative strength is used for MID_CPLP here in form of theorem 2 below.

Result 3:

$$
C P L P{ }_{-} R 1\left(\lambda 3_{j}\right)+O F V_{-} 0-1_{-} K S P\left(\lambda 3_{j}\right)
$$

$$
\leq C P L P_{-} R 2\left(\lambda 3_{j}\right)+O F V_{-} 0-1_{-} K S P\left(\lambda 3_{j}\right) \leq C P L P P_{-} R 3\left(\lambda 3_{j}\right)+O F V_{-} 0-1_{-} K S P\left(\lambda 3_{j}\right) .
$$

where OFV stands for objective function value of $0-1_{-} K S P\left(\lambda 3_{j}\right)$. Hence we have

Theorem 1: For any value of $\left(\lambda 3_{j}\right)$,

$M I D_{-} C P L P_{-} R 1\left(\lambda 3_{j}\right) \leq M I D_{-} C P L P_{-} R 2\left(\lambda 3_{j}\right) \leq M I D_{-} C P L P \_R 3\left(\lambda 3_{j}\right)$.

This theorem provides the relative effectiveness of the bounds that may be obtained for these relaxations of MID_CPLP. Verma and Sharma [5] have shown that difference between R2-R1 and R3-R2 is significant. Hence we have the result given below.

Result 4: Difference between $M I D_{-} C P L P_{-} R 2\left(\lambda 3_{j}\right)$ and $M I D_{-} C P L P P_{-} R 1\left(\lambda 3_{j}\right)$ \& $M I D_{-} C P L P P_{-} R 3\left(\lambda 3_{j}\right)$ and $M I D_{-} C P L P_{-} R 2\left(\lambda 3_{j}\right)$ is significant.

Now we add two new constraints to the MID_CPLP. They are as follows.

$$
x w s 1 w s 2_{i j} \leq \operatorname{capws}_{i} y w s 2_{j}, \forall i, j,
$$




$$
x w s 1 w s 2_{i j} \leq \operatorname{capws}_{j} y w s 1_{i}, \forall i, j,
$$

We get the modified and a valid formulation of MID_CPLP i.e., MO_MID_CPLP as given below.

\subsubsection{MO_MID_CPLP}

$$
\begin{aligned}
Z_{\text {MO_MID_CPLP }} & =f_{2}\left(x w s 1 w s 2_{i j},\left(c w s 1 w s 2_{i j}-\lambda 1_{i}+\lambda 2_{j}\right), y w s 1_{i}, y w s 2_{j}, f w s 1_{i}, f w s 2_{j}\right) \\
& =\min _{x w s 1 w s 2, y w s 1, y w s 2} \sum_{i, j}\left(c w s 1 w s 2_{i j}-\lambda 1_{i}+\lambda 2_{j}\right) * x w s 1 w s 2_{i j}+\frac{1}{2} \sum_{i} y w s 1_{i} * f w s 1_{i}+\frac{1}{2} \sum_{j} y w s 2_{j} * f w s 2_{j}
\end{aligned}
$$

s.t.: (2(a)), (5(a-i)), (5(a-ii)), (6(a-i)), (6(a-ii)), (7(a)), (8(a)), (9(a)), (10(a)), (8(b)), (9(b)), (10(b)), (14) and (15).

Now we relax the constraints (5(a-ii)), (14) and (15) by attaching multiplier $\lambda 3_{j}, \lambda 4_{i j}$ and $\lambda 5_{i j}$ respectively to get the following.

1) $M O \_M I D \_C P L P \_R\left(23_{j}, \lambda 4_{i j}, \lambda 5_{i j}\right)$

$$
\begin{aligned}
Z_{\text {MO_MID_CPLP_R }}= & \min _{x w s 1 w s 2, y w s 1, y w s 2} \sum_{i, j}\left(c w s 1 w s 2_{i j}-\lambda 1_{i}+\lambda 2_{j}+\lambda 3_{j}+\lambda 4_{i j}+\lambda 5_{i j}\right) * x w s 1 w s 2_{i j} \\
& +\frac{1}{2} \sum_{i} y w s 1_{i} * f w s 1_{i}-\sum_{i, j} \text { capws } 2_{j} * y w s 1_{i} * \lambda 5_{i, j}
\end{aligned}
$$

s.t.: (2(a)), (5(a-i)), (6(a-i)), (6(a-ii)), (7(a)), (8(a)), (9(a)), (10(a)), (10(b)).

2) MO_0-1_KSP $\left(\lambda 3_{j}, \lambda 4_{i j}, \lambda 5_{i j}\right)$

$$
Z_{M O_{-} 0-1 \_K S P}=\frac{1}{2} \sum_{j} y w s 2_{j} * f w s 2_{j}-\sum_{j} c a p w s 2_{j} * y w s 2_{j} * \lambda 3_{j}-\sum_{i, j} c a p w s 1_{i} * y w s 2_{j} * \lambda 4_{i, j}
$$

s.t.: (8(b)), (9(b)).

\section{3) Relaxation of MO_MID_CPLP_R $\left(\lambda 3_{i}, \lambda 4_{i j}, \lambda 5_{i j}\right)$}

Now MO_MID_CPLP_R $\left(\lambda 3_{j}, \lambda 4_{j}, \lambda 5_{j}\right)$ can be attempted by three different relaxations. They are $R 1, R 2$ and $R 3$ respectively as before.

$$
\begin{aligned}
\text { Result 5: We get } & M O_{-} M I D_{-} C P L P_{-} R 1\left(\lambda 3_{j}^{*}, \lambda 4_{i, j}^{*}, \lambda 5_{i, j}^{*}\right) \leq M O_{-} M I D_{-} C P L P \_R 2\left(\lambda 3_{j}^{*}, \lambda 4_{i, j}^{*}, \lambda 5_{i, j}^{*}\right) . \\
& \leq M O_{-} M I D_{-} C P L P_{-} R 3\left(\lambda 3_{j}^{*}, \lambda 4_{i, j}^{*}, \lambda 5_{i, j}^{*}\right)
\end{aligned}
$$

Theorem 2: For optimal value of $\left(\lambda 3_{j}^{*}, \lambda 4_{i, j}^{*}, \lambda 5_{i, j}^{*}\right)$,

$$
\begin{aligned}
& M O_{-} M I D \_C P L P \_R 1\left(\lambda 3_{j}^{*}, \lambda 4_{i, j}^{*}, \lambda 5_{i, j}^{*}\right) \leq M O_{-} M I D_{-} C P L P \_R 2\left(\lambda 3_{j}^{*}, \lambda 4_{i, j}^{*}, \lambda 5_{i, j}^{*}\right) . \\
& \leq M O_{-} M I D_{-} C P L P \_R 3\left(\lambda 3_{j}^{*}, \lambda 4_{i, j}^{*}, \lambda 5_{i, j}^{*}\right)
\end{aligned}
$$

Result 6: Differences between $M O \_M I D \_C P L P \_R 2$ and $M O \_M I D \_C P L P \_R 1 \& M O \_M I D \_C P L P \_R 3$ and $M O \_M I D \_C P L P \_R 2$ are statistically significant.

Now since $R 1$ is $L P$ relaxation, it easily follows that

Theorem 3: $C P L P_{-} R 1\left(\lambda 3_{j}^{*}\right) \leq M O_{-} M I D_{-} C P L P_{-} R 1\left(\lambda 3_{j}^{*}, \lambda 4_{i, j}^{*}, \lambda 5_{i, j}^{*}\right)$.

In R2: we solve single constraint bounded variable LP to get a factor that is added to $0-1$ single constraint knapsack problem (which is solved by inspection) (see Christifides and Beasley [15]). A priori it is observed that the process is complicated enough and is NOT amenable to theoretical result (for determining the difference between MID_CPLP $(R 2)$ and $M O \_M I D \_C P L P(R 2)$.

In R3: we solve several continuous knapsack problems \& their objective function value is fed to a $0-1$ knapsack problem (see Nauss [16]). This is also complicated enough; and theoretical result may NOT be possible (for determining the difference between MID_CPLP (R3) and MO_MID_CPLP (R3)).

Hence, we may have to determine differences empirically (by coding $R 2$ and $R 3$ for different Lagrangian Relaxations). However, as warehouse capacities are more constrained, MO_MID_CPLP for both $R 2$ and $R 3$ will give significantly superior lagrangian lower bounds at little computational expense. However, we do have a 
theoretical result for R1 (for difference between MID_CPLP and MO_MID_CPLP) which a LP relaxation. Hence we have the following Conjectures.

Conjecture 1: $C P L P_{-} R 2\left(\lambda 3_{j}^{*}\right) \leq M O_{-} C P L P_{-} R 2\left(\lambda 3_{j}^{*}, \lambda 4_{i, j}^{*}, \lambda 5_{i, j}^{*}\right)$.

Conjecture 2: $C P L P_{-} R 3\left(\lambda 3_{j}^{*}\right) \leq M O_{-} C P L P_{-} R 3\left(\lambda 3_{j}^{*}, \lambda 4_{i, j}^{*}, \lambda 5_{i, j}^{*}\right)$.

If only (14) is added to MID_CPLP then we get MO1_MID_CPLP. Several similar results are given without much discussion as they are obvious.

\subsubsection{MO1_MID_CPLP}

$$
\begin{aligned}
Z_{\text {MO1_MID_CPLP }} & =f_{2}\left(x w s 1 w s 2_{i j},\left(c w s 1 w s 2_{i j}-\lambda 1_{i}+\lambda 2_{j}, y w s 1_{i}, y w s 2_{j}, f w s 1_{i}, f w s 2_{j}\right)\right) \\
& =\min _{\text {xws } 1 w s 2, y w s 1, y w s 2} \sum_{i, j}\left(c w s 1 w s 2_{i j}-\lambda 1_{i}+\lambda 2_{j}\right) * x w s 1 w s 2_{i j}+\frac{1}{2} \sum_{i} y w s 1_{i} * f w s 1_{i}+\frac{1}{2} \sum_{j} y w s 2_{j} * f w s 2_{j}
\end{aligned}
$$

s.t.: (2(a)), (5(a-i)), (5(a-ii)), (6(a-i)), (6(a-ii)), (7(a)), (8(a)), (9(a)), (10(a)), (8(b)), (9(b)), (10(b)), (14).

Now we relax the constraints (5(a-ii)) and (14) by attaching multiplier $\lambda 3_{j}$ and $\lambda 4_{i j}$ respectively to get the following.

1) $M O 1_{-} M I D_{-} C P L P \_R\left(\lambda 3_{j}, \lambda 4_{i j}\right)$

$$
\begin{aligned}
& Z_{\text {MO1_MID_CPLP_R }} \\
& =\min _{x w s 1 w s 2, y w s 1, y w s 2} \sum_{i, j}\left(c w s 1 w s 2_{i j}-\lambda 1_{i}+\lambda 2_{j}+\lambda 3_{j}+\lambda 4_{i j}\right) * x w s 1 w s 2_{i j}+\frac{1}{2} \sum_{i} y w s 1_{i} * f w s 1_{i}
\end{aligned}
$$

s.t.: (2(a)), (5(a-i)), (6(a-i)), (6(a-ii)), (7(a)), (8(a)), (9(a)), (10(a)), (10(b)).

2) $M O 1_{-} 0-1 \_K S P\left(\lambda 3_{j}, \lambda 4_{i j}\right)$

$$
Z_{\text {MO1_0-1_KSP }_{-}}=\frac{1}{2} \sum_{j} y w s 2_{j} * f w s 2_{j}-\sum_{j} \operatorname{capws} 2_{j} * y w s 2_{j} * \lambda 3_{j}-\sum_{i, j} \operatorname{capws} 1_{i} * y w s 2_{j} * \lambda 4_{i, j}
$$

s.t.: (8(b)), (9(b)).

3) Relaxation of $M O 1_{-} M I D_{-} C P L P_{-} R\left(\lambda 3_{j}, \lambda 4_{i j}\right)$

Now MO1_MID_CPLP_R $\left(\lambda 3_{j}, \lambda 4_{i j}\right)$ can be attempted by three different relaxations. They are R1, R2 and $\mathrm{R} 3$ respectively as before.

Result 7: We get

$$
\begin{aligned}
& M O 1_{-} M I D_{-} C P L P \_R 1\left(\lambda 3_{j}^{*}, \lambda 4_{i, j}^{*}\right) \leq M O 1_{-} M I D_{-} C P L P P_{-} R 2\left(\lambda 3_{j}^{*}, \lambda 4_{i, j}^{*}\right) . \\
& \leq M O 1_{-} M I D_{-} C P L P \_R 3\left(\lambda 3_{j}^{*}, \lambda 4_{i, j}^{*}\right)
\end{aligned} .
$$

Theorem 4: For optimal value of $\left(\lambda 3_{j}^{*}, \lambda 4_{i, j}^{*}\right)$,

$M O 1_{-} M I D_{-} C P L P \_R 1\left(\lambda 3_{j}^{*}, \lambda 4_{i, j}^{*}\right) \leq M O 1_{-} M I D_{-} C P L P P_{-} R 2\left(\lambda 3_{j}^{*}, \lambda 4_{i, j}^{*}\right)$.

$\leq M O 1_{-} M I D_{-} C P L P \_R 3\left(\lambda 3_{j}^{*}, \lambda 4_{i, j}^{*}\right)$

Result 8: Differences between $M O 1 \_M I D \_C P L P \_R 2$ and $M O 1 \_M I D \_C P L P \_R 1$ \& MO1_MID_CPLP_R3 and MO1_MID_CPLP_R2 are statistically significant.

Now since R1 is LP relaxation, it easily follows that

Theorem 5: $C P L P P_{-} R\left(\lambda 3_{j}^{*}\right) \leq M O 1_{-} M I D_{-} C P L P_{-} R 1\left(\lambda 3_{j}^{*}, \lambda 4_{i, j}^{*}\right)$.

Conjecture 3: $C P L P_{-} R 2\left(\lambda 3_{j}^{*}\right) \leq M O 1_{-} C P L P_{-} R 2\left(\lambda 3_{j}^{*}, \lambda 4_{i, j}^{*}\right)$.

Conjecture 4: $C P L P_{-} R 3\left(\lambda 3_{j}^{*}\right) \leq M O 1_{-} C P L P_{-} R 3\left(\lambda 3_{j}^{*}, \lambda 4_{i, j}^{*}\right)$.

If only (15) is added to MID_CPLP then we get, MO2_MID_CPLP. 


\subsubsection{MO2_MID_CPLP}

$$
\begin{aligned}
Z_{M O 2_{-} M I D_{-} C P L P}= & f_{2}\left(x w s 1 w s 2_{i j},\left(c w s 1 w s 2_{i j}-\lambda 1_{i}+\lambda 2_{j}\right), y w s 1_{i}, y w s 2_{j}, f w s 1_{i}, f w s 2_{j}\right) \\
= & \min _{x w s 1 w s 2, y w s 1, y w s 2} \sum_{i, j}\left(c w s 1 w s 2_{i j}-\lambda 1_{i}+\lambda 2_{j}\right) * x w s 1 w s 2_{i j}+\frac{1}{2} \sum_{i} y w s 1_{i} * f w s 1_{i} \\
& +\frac{1}{2} \sum_{j} y w s 2_{j} * f w s 2_{j}
\end{aligned}
$$

s.t.: (2(a)), (5(a-i)), (5(a-ii)), (6(a-i)), (6(a-ii)), (7(a)), (8(a)), (9(a)), (10(a)), (8(b)), (9(b)), (10(b)), and (15).

Now we relax the constraints (5(a-ii)) and (15) by attaching multiplier $\lambda 3_{j}$ and $\lambda 5_{i j}$ respectively to get the following.

1) $M O 2_{-} M I D D_{C} C P L P \_R\left(\lambda 3_{j}, \lambda 5_{i j}\right)$

$$
\begin{aligned}
Z_{\text {MO2_MID_CPLP_R }}= & \operatorname{mins}_{\text {xws } 2, y w s, y w s 2} \sum_{i, j}\left(c w s 1 w s 2_{i j}-\lambda 1_{i}+\lambda 2_{j}+\lambda 3_{j}+\lambda 5_{i j}\right) * x w s 1 w s 2_{i j} \\
& +\frac{1}{2} \sum_{i} y w s 1_{i} * f w s 1_{i}-\sum_{i, j} c a p w s 2_{j} * y w s 1_{i} * \lambda 5_{i, j}
\end{aligned}
$$

s.t.: (2(a)), (5(a-i)), (6(a-i)), (6(a-ii)), (7(a)), (8(a)), (9(a)), (10(a)), (10(b)).

2) $M O 2_{-} 0-1_{-} \operatorname{KSP}\left(\lambda 3_{j}, \lambda 5_{i j}\right)$

$$
Z_{\mathrm{MO} Z_{-} 0-1 \_K S P}=\frac{1}{2} \sum_{j} y w s 2_{j} * f w s 2_{j}-\sum_{j} c a p w s 2_{j} * y w s 2_{j} * \lambda 3_{j}
$$

s.t.: (8(b)), (9(b)).

3) Relaxation of $M O 2_{-} M I D_{-} C P L P \_R\left(\lambda 3_{j}, \lambda 5_{i j}\right)$

Now MO2_MID_CPLP $2_{-} R\left(\lambda 3_{j}, \lambda 5_{i j}\right)$ is attempted in literature by three different relaxations. They are R1, R2 and R3 respectively as before. When integrality restriction on yws1 $1_{i}$ is relaxed we obtain (12(a)).

Result 9: we get,

$$
\begin{aligned}
& M O 2 \_M I D_{-} C P L P \_R 1\left(\lambda 3_{j}^{*}, \lambda 5_{i, j}^{*}\right) \leq M O 2_{-} M I D_{-} C P L P \_R 2\left(\lambda 3_{j}^{*}, \lambda 5_{i, j}^{*}\right) . \\
& \leq M O 2 \_M I D_{-} C P L P_{-} R 3\left(\lambda 3_{j}^{*}, \lambda 5_{i, j}^{*}\right)
\end{aligned} .
$$

Theorem 6: For optimal value of $\left(\lambda 3_{j}^{*}, \lambda 5_{i, j}^{*}\right)$,

$$
M O 2_{-} M I D{ }_{-} C P L P P_{-} R 1\left(\lambda 3_{j}^{*}, \lambda 5_{i, j}^{*}\right) \leq M O 2_{-} M I D_{-} C P L P P_{-} R 2\left(\lambda 3_{j}^{*}, \lambda 5_{i, j}^{*}\right) \text {. }
$$

$\leq M O 2_{-} M I D_{-} C P L P \_R 3\left(\lambda 3_{j}^{*}, \lambda 5_{i, j}^{*}\right)$

Result 10: Differences between $M O 2 \_M I D \_C P L P \_R 2$ and $M O 2 \_M I D \_C P L P \_R 1$ \& $M O 2 \_M I D \_C P L P \_R 3$ and MO2_MID_CPLP_R2 are statistically significant.

Now since R1 is LP relaxation, it easily follows that

Theorem 7: $C P L P P_{-} R 1\left(\lambda 3_{j}^{*}\right) \leq M O_{-} M I D_{-} C P L P_{-} R 1\left(\lambda 3_{j}^{*}, \lambda 5_{i, j}^{*}\right)$.

Conjecture 5: $C P L P \_R 2\left(\lambda 3_{j}^{*}\right) \leq M O 2_{-} C P L P \_R 2\left(\lambda 3_{j}^{*}, \lambda 5_{i, j}^{*}\right)$..

Conjecture 6: $C P L P_{-} R 3\left(\lambda 3_{j}^{*}\right) \leq M O 2_{-} C P L P P_{-} R 3\left(\lambda 3_{j}^{*}, \lambda 5_{i, j}^{*}\right)$.

By the similar logic we have

Theorem 8: we have, $M O 1_{-} C P L P_{-} R 1\left(\lambda 3_{j}^{*}, \lambda 4_{i, j}^{*}\right) \leq M O_{-} C P L P \_R 1\left(\lambda 3_{j}^{*}, \lambda 4_{i, j}^{*}, \lambda 5_{i, j}^{*}\right)$.

Theorem 9: we have, $M O 2_{-} C P L P_{-} R 1\left(\lambda 3_{j}^{*}, \lambda 5_{i, j}^{*}\right) \leq M O_{-} C P L P_{-} R 1\left(\lambda 3_{j}^{*}, \lambda 4_{i, j}^{*}, \lambda 5_{i, j}^{*}\right)$.

Now we give two more conjectures that are based on similar logic given earlier.

Conjecture 7: $C P L P_{-} R 2\left(\lambda 3_{j}^{*}, \lambda 5_{i, j}^{*}\right) \leq M O_{-} C P L P_{-} R 2\left(\lambda 3_{j}^{*}, \lambda 4_{i, j}^{*}, \lambda 5_{i, j}^{*}\right)$.

Conjecture 8: $C P L P_{-} R 3\left(\lambda 3_{j}^{*}, \lambda 4_{i, j}^{*}\right) \leq M O 2_{-} C P L P P_{-} R 3\left(\lambda 3_{j}^{*}, \lambda 4_{i, j}^{*}, \lambda 5_{i, j}^{*}\right)$.

Since the effect of $\lambda 4_{i j}$ and $\lambda 5_{i j}$ is known only after it is through two combinatorial optimization problems, we believe a priori that it will difficult theoretically to prove the conjectures given in this paper. Hence we propose to carryout empirical investigation to support these conjectures. 


\section{Empirical Investigation and Statistical Significance}

We have created sample problems randomly for the TSCWLP problem of sizes $25 \times 25 \times 25,50 \times 50 \times 50,75 \times$ $75 \times 75$, and $100 \times 100 \times 100:$ CPLP_R1, CPLP_R2, CPLP_R3 and KSP; MO_CPLP_R1, MO_CPLP_R2, MO_CPLP_R3 and MO_KSP; MO1_CPLP_R1, MO1_CPLP_R2, MO1_CPLP_R3 and MO1_KSP; MO2_CPLP_R1, MO2_CPLP_R2, MO2_CPLP_R3 and MO2_KSP for the different values of $\theta$ such as $2,1.6$ and 1.2. For each category of the problem size we created 25 problems for fws1(i): 111-999, fws2(j): 111-999, capws1(i): 0.111-0.999 such that sum capws1(i) $\geq 1$, capws2(j): 0.111-0.999 such that sum capws2(j) $\geq 1$, cws1ws2(i,j): 1111-9999, thus created 3600 problem instances. We used the CPLEX 12.4.0.0 solver of GAMS 23.8 on an Intel (R) Core (TM) i7 CPU 870@2.93GHz and 4 GB RAM, 64 bit operating system to solve the problem instances. For each problem categories, we applied Lagrangian relaxations. Here, we obtained after Lagrangian Relaxation CPLP_R: Objective Function value of CPLP_R, KSP: Objective Function value of 0-1 Knapsack, MID_CPLP_R: Objective Function value of MID_CPLP_R, for each type of relaxations for R1, R2 and R3. Similar details for MO, MO1 and MO2 for R1, R2 and R3 can be written. Here, we only concentrate upon the objective function values of CPLP_R, KSP and MID_CPLP_R by applying Lagrangian Relaxations on the different formulations. After taking maximum objective function values of CPLP_R, KSP and MID_ CPLP_R for the different values of $\theta$ (denoted by ${ }^{*}$ ) for each category. The results of statistical significance are reported in the Table 1 , Table 2, Table 3 and Table 4 . In Table 5, standard t-critical values are given for $n=25$, $\mathrm{df}=24$. Table 6 is giving all the information about empirical investigation.

\section{Conclusion}

Here we attempt problem TSCWLP by vertical decomposition. When the flow balance equations are relaxed, it leads to three versions of CPLP; that is, RHS_CPLP, LHS_CPLP and MID_CPLP. RHS_CPLP has been solved by Cornuejol, Sridharan and Thizy [14] and LHS_CPLP has been solved by Verma and Sharma [5]. We solve MID_CPLP again by LR and give interesting theoretical results; and these were mostly verified by empirical

Table 1. T-stat values for difference in performance of Lagrangian Relaxations on Model(j) - Model(i).

\begin{tabular}{|c|c|c|c|c|c|c|}
\hline \multirow{2}{*}{$\begin{array}{c}\text { No. of } \\
\text { warehouses at } \\
\text { stage } 1 * \text { stage } 2\end{array}$} & \multicolumn{2}{|r|}{ Problem Category } & \multicolumn{2}{|r|}{ R2(i) } & \multicolumn{2}{|r|}{ R3(i) } \\
\hline & & & CPLP_R2* & (CPLP_R2+KSP_R2)* & CPLP_R3* ${ }^{*}$ & (CPLP_R3+KSP_R3)* \\
\hline \multirow{4}{*}{$\begin{array}{c}100 * 100 \\
\mathrm{n}=25\end{array}$} & \multirow{2}{*}{$\mathrm{R} 1(\mathrm{j})$} & CPLP_R1* & $3.71^{* * *}$ & & $2.96^{* * *}$ & \\
\hline & & $($ CPLP_R1+KSP_R1) & & $2.86^{* * *}$ & & $2.06^{* *}$ \\
\hline & \multirow{2}{*}{$\mathrm{R} 2(\mathrm{j})$} & CPLP_R2 ${ }^{*}$ & & & $-2.05^{* *}$ & \\
\hline & & (CPLP_R2+KSP_R2) ${ }^{*}$ & & & & $-2.06^{* *}$ \\
\hline \multirow{4}{*}{$\begin{array}{l}75 * 75 \\
\mathrm{n}=25\end{array}$} & \multirow{2}{*}{$\mathrm{R} 1(\mathrm{j})$} & CPLP_R1 ${ }^{*}$ & $2.88^{* * *}$ & & $2.88^{* * *}$ & \\
\hline & & (CPLP_R1+KSP_R1) ${ }^{*}$ & & $1.33^{*}$ & & $1.33^{*}$ \\
\hline & \multirow{2}{*}{$\mathrm{R} 2(\mathrm{j})$} & CPLP_R2 ${ }^{*}$ & & & $-1.30^{*}$ & \\
\hline & & (CPLP_R2+KSP_R2) & & & & $-1.30^{*}$ \\
\hline \multirow{4}{*}{$\begin{array}{l}50 * 50 \\
\mathrm{n}=25\end{array}$} & \multirow{2}{*}{$\mathrm{R} 1(\mathrm{j})$} & CPLP_R1 ${ }^{*}$ & $2.01^{* *}$ & & 1.12 & \\
\hline & & $($ CPLP_R1+KSP_R1) & & $1.58^{*}$ & & 1.07 \\
\hline & \multirow{2}{*}{$\mathrm{R} 2(\mathrm{j})$} & CPLP_R2 ${ }^{*}$ & & & 0.99 & \\
\hline & & $\left(\right.$ CPLP_R2+KSP_R2) ${ }^{*}$ & & & & 0.99 \\
\hline \multirow{4}{*}{$\begin{array}{l}25 * 25 \\
n=25\end{array}$} & \multirow{2}{*}{$\mathrm{R} 1(\mathrm{j})$} & CPLP_R1 ${ }^{*}$ & 1.21 & & 1.02 & \\
\hline & & $\left(\right.$ CPLP_R1+KSP_R1) ${ }^{*}$ & & 0.91 & & 1.02 \\
\hline & \multirow{2}{*}{$\mathrm{R} 2(\mathrm{j})$} & CPLP_R2 ${ }^{*}$ & & & 1.00 & \\
\hline & & $\left(\right.$ CPLP_R2+KSP_R2) ${ }^{*}$ & & & & 1.03 \\
\hline
\end{tabular}

${ }^{* * *}$ Significant at 0.005 level, ${ }^{* *}$ Significant at 0.05 level, ${ }^{*}$ Significant at 0.10 level. CPLP_R1 ${ }^{*}$, CPLP_R2 ${ }^{*}$, CPLP_R3 ${ }^{*}$, (CPLP_R1+KSP_R1) ${ }^{*}$, (CPLP_R2+KSP_R2) $^{*}$ and (CPLP_R3+KSP_R3) ${ }^{*}$ are the maximum values of CPLP_R1, CPLP_R2, CPLP_R3, (CPLP_R1+KSP_R1), (CPLP_R2+KSP_R2) and (CPLP_R3+KSP_R3). 
Table 2. T-stat values for difference in performance of Lagrangian Relaxations on Model(j) - Model(i).

\begin{tabular}{|c|c|c|c|c|c|c|}
\hline \multirow{2}{*}{$\begin{array}{c}\text { No. of } \\
\text { warehouses at } \\
\text { stage } 1^{*} \text { stage } 2\end{array}$} & \multicolumn{2}{|c|}{ Problem Category } & \multicolumn{2}{|r|}{ MO_R2(i) } & \multicolumn{2}{|r|}{ MO_R3(i) } \\
\hline & Pro & Dien Category & CPLP_R2* & (CPLP_R2+KSP_R2) ${ }^{*}$ & CPLP_R3* & (CPLP_R3+KSP_R3)* \\
\hline \multirow{4}{*}{$\begin{array}{c}100 * 100 \\
\mathrm{n}=25\end{array}$} & \multirow{2}{*}{ MO_R1(j) } & CPLP_R1 ${ }^{*}$ & $-5.64^{* * *}$ & & $-11.67^{* * *}$ & \\
\hline & & (CPLP_R1+KSP_R1)* & & $-5.61^{* * *}$ & & $-11.63^{* * *}$ \\
\hline & \multirow{2}{*}{ MO_R2(j) } & CPLP_R2 ${ }^{*}$ & & & $-3.51^{* * *}$ & \\
\hline & & $\left(\right.$ CPLP_R2+KSP_R2) ${ }^{*}$ & & & & $-3.50^{* * *}$ \\
\hline \multirow{4}{*}{$\begin{array}{l}75 * 75 \\
n=25\end{array}$} & \multirow{2}{*}{ MO_R1(j) } & CPLP_R1 ${ }^{*}$ & $-5.15^{* * *}$ & & $-12.22^{* * *}$ & \\
\hline & & (CPLP_R1+KSP_R1) ${ }^{*}$ & & $-2.25^{* *}$ & & $-4.46^{* * *}$ \\
\hline & \multirow{2}{*}{ MO_R2(j) } & CPLP_R2 $^{*}$ & & & $-2.73^{* *}$ & \\
\hline & & (CPLP_R2+KSP_R2) & & & & -0.04 \\
\hline \multirow{4}{*}{$\begin{array}{l}50 * 50 \\
n=25\end{array}$} & \multirow{2}{*}{ MO_R1(j) } & CPLP_R1 ${ }^{*}$ & $-1.69^{*}$ & & 0.47 & \\
\hline & & $\left(\right.$ CPLP_R1+KSP_R1) ${ }^{*}$ & & $-1.59^{*}$ & & 0.21 \\
\hline & \multirow{2}{*}{ MO_R2(j) } & CPLP_R2* & & & 0.91 & \\
\hline & & $\left(\right.$ CPLP_R2+KSP_R2) ${ }^{*}$ & & & & 0.57 \\
\hline \multirow{4}{*}{$\begin{array}{l}25 * 25 \\
\mathrm{n}=25\end{array}$} & \multirow{2}{*}{ MO_R1(j) } & CPLP_R1 ${ }^{*}$ & 0.45 & & 0.93 & \\
\hline & & $\left(\mathrm{CPLP} \_\mathrm{R} 1+\mathrm{KSP} \_\mathrm{R} 1\right)^{*}$ & & 0.47 & & 0.93 \\
\hline & \multirow{2}{*}{ MO_R2(j) } & CPLP_R2* & & & 0.98 & \\
\hline & & $\left(\right.$ CPLP_R2+KSP_R2) ${ }^{*}$ & & & & 0.98 \\
\hline
\end{tabular}

\begin{abstract}
${ }^{* * *}$ Significant at 0.005 level, ${ }^{* *}$ Significant at 0.05 level, ${ }^{*}$ Significant at 0.10 level. CPLP_R1 ${ }^{*}$, CPLP_R2 ${ }^{*}$, CPLP_R3 ${ }^{*}$, (CPLP_R1+KSP_R1) ${ }^{*}$, (CPLP_R2+KSP_R2) ${ }^{*}$ and (CPLP_R3+KSP_R3) ${ }^{*}$ are the maximum values of CPLP_R1, CPLP_R2, CPLP_R3, (CPLP_R1+KSP_R1),
\end{abstract} (CPLP_R2+KSP_R2) and (CPLP_R3+KSP_R3).

Table 3. T-stat values for difference in performance of Lagrangian Relaxations on Model(j) - Model(i).

\begin{tabular}{|c|c|c|c|c|c|c|}
\hline \multirow{2}{*}{$\begin{array}{c}\text { No. of } \\
\text { warehouses at } \\
\text { stage } 1 * \text { stage } 2\end{array}$} & \multicolumn{2}{|c|}{ Problem Category } & \multicolumn{2}{|r|}{ MO1_R2(i) } & \multicolumn{2}{|r|}{ MO1_R3(i) } \\
\hline & Fro & Jiem Category & CPLP_R2* & (CPLP_R2+KSP_R2) ${ }^{*}$ & CPLP_R3* & (CPLP_R3+KSP_R3)* \\
\hline \multirow{4}{*}{$\begin{array}{c}100 * 100 \\
n=25\end{array}$} & \multirow[b]{2}{*}{ MO1_R1(j) } & CPLP_R1 ${ }^{*}$ & $-7.86^{* * *}$ & & $-16.26^{* * *}$ & \\
\hline & & $($ CPLP_R1+KSP_R1) & & $-7.53^{* * *}$ & & $-16.25^{* * *}$ \\
\hline & \multirow{2}{*}{ MO1_R2(j) } & CPLP_R2* ${ }^{*}$ & & & $5.65^{* * *}$ & \\
\hline & & $\left(\right.$ CPLP_R2+KSP_R2) ${ }^{*}$ & & & & $5.59^{* * * *}$ \\
\hline \multirow{4}{*}{$\begin{array}{l}75 * 75 \\
\mathrm{n}=25\end{array}$} & \multirow{2}{*}{ MO1_R1(j) } & CPLP_R1* & $-8.20^{* * *}$ & & $-14.47^{* * *}$ & \\
\hline & & $\left(\right.$ CPLP_R1+KSP_R1) ${ }^{*}$ & & $-3.02^{* * *}$ & & $-14.74^{* * *}$ \\
\hline & \multirow{2}{*}{ MO1_R2(j) } & CPLP_R2 ${ }^{*}$ & & & $-2.78^{* *}$ & \\
\hline & & $\left(\right.$ CPLP_R2+KSP_R2) ${ }^{*}$ & & & & 0.62 \\
\hline \multirow{4}{*}{$\begin{array}{l}50 * 50 \\
\mathrm{n}=25\end{array}$} & \multirow{2}{*}{ MO1_R1(j) } & CPLP_R1 ${ }^{*}$ & $-2.89^{* * *}$ & & 0.31 & \\
\hline & & $\left(\right.$ CPLP_R1+KSP_R1) ${ }^{*}$ & & $-2.88^{* * *}$ & & 0.31 \\
\hline & \multirow{2}{*}{ MO1_R2(j) } & $\mathrm{CPLP}_{\mathrm{N}} 2^{*}$ & & & 0.92 & \\
\hline & & (CPLP_R2+KSP_R2) ${ }^{*}$ & & & & 0.92 \\
\hline \multirow{4}{*}{$\begin{array}{l}25 * 25 \\
\mathrm{n}=25\end{array}$} & \multirow{2}{*}{ MO1_R1(j) } & CPLP_R1 ${ }^{*}$ & 0.16 & & 0.90 & \\
\hline & & $\left(\right.$ CPLP_R1+KSP_R1) ${ }^{*}$ & & 0.17 & & 0.90 \\
\hline & \multirow{2}{*}{ MO1_R2(j) } & CPLP_R2* & & & 0.99 & \\
\hline & & (CPLP_R2+KSP_R2) $^{*}$ & & & & 0.98 \\
\hline
\end{tabular}

${ }^{* * *}$ Significant at 0.005 level, ${ }^{* *}$ Significant at 0.05 level, "Significant at 0.10 level. CPLP_R1 ${ }^{*}$, CPLP_R2 ${ }^{*}$, CPLP_R3 ${ }^{*}$, (CPLP_R1+KSP_R1) (CPLP_R2+KSP_R2) $^{*}$ and (CPLP_R3+KSP_R3) ${ }^{*}$ are the maximum values of CPLP_R1, CPLP_R2, CPLP_R3, (CPLP_R1+KSP_R1), (CPLP_R2+KSP_R2) and (CPLP_R3+KSP_R3). 
Table 4. T-stat values for difference in performance of Lagrangian Relaxations on Model(j) - Model(i).

\begin{tabular}{|c|c|c|c|c|c|c|}
\hline \multirow{2}{*}{$\begin{array}{c}\text { No. of } \\
\text { warehouses at } \\
\text { stage } 1 * \text { stage } 2\end{array}$} & \multicolumn{2}{|c|}{ Problem Category } & \multicolumn{2}{|r|}{ MO2_R2(i) } & \multicolumn{2}{|c|}{ MO2_R3(i) } \\
\hline & Pro & Hemi Category & CPLP_R2* & CPLP_R2 ${ }^{*}+$ KSP_R2* & CPLP_R3* & CPLP_R3 ${ }^{*}+$ KSP_R3 ${ }^{*}$ \\
\hline \multirow{4}{*}{$100 * 100$} & \multirow{2}{*}{ MO2_R1(j) } & CPLP_R1* ${ }^{*}$ & $-1.78^{* *}$ & & $-7.60^{* * *}$ & \\
\hline & & CPLP_R1 ${ }^{*}+K S P \_R 1^{*}$ & & -1.02 & & $-3.61^{* * *}$ \\
\hline & \multirow{2}{*}{ MO2_R2(j) } & CPLP_R2 ${ }^{*}$ & & & $-3.35^{* * *}$ & \\
\hline & & CPLP_R2 ${ }^{*}+$ KSP_R2* & & & & 0.59 \\
\hline \multirow{4}{*}{$75 * 75$} & \multirow{2}{*}{ MO2_R1(j) } & CPLP_R1 ${ }^{*}$ & -0.96 & & $-7.70^{* * * *}$ & \\
\hline & & CPLP_R $1^{*}+\mathrm{KSP} R 1^{*}$ & & 0.66 & & 0.17 \\
\hline & \multirow{2}{*}{ MO2_R2(j) } & CPLP_R2 ${ }^{*}$ & & & $-2.48^{* *}$ & \\
\hline & & CPLP_R2 ${ }^{*}+$ KSP_R2* & & & & $-2.48^{* *}$ \\
\hline \multirow{4}{*}{$50 * 50$} & \multirow{2}{*}{ MO2_R1(j) } & CPLP_R1 ${ }^{*}$ & 0.45 & & 0.84 & \\
\hline & & CPLP_R1 ${ }^{*}+K S P \_R 1^{*}$ & & 0.45 & & 0.84 \\
\hline & & CPLP_R2* ${ }^{*}$ & & & 0.89 & \\
\hline & MIUL_R2(J) & CPLP_R2 ${ }^{*}+$ KSP_R2* & & & & 0.89 \\
\hline \multirow{4}{*}{$25 * 25$} & \multirow{2}{*}{ MO2_R1(j) } & CPLP_R1 ${ }^{*}$ & 0.98 & & 0.98 & \\
\hline & & CPLP_R $1^{*}+K S P \_R 1^{*}$ & & 0.68 & & 0.98 \\
\hline & \multirow{2}{*}{ MO2_R2(j) } & CPLP_R2 ${ }^{*}$ & & & 0.98 & \\
\hline & & CPLP_R2 ${ }^{*}+$ KSP_R2* & & & & 1.01 \\
\hline
\end{tabular}

${ }^{* * *}$ Significant at 0.005 level, ${ }^{* *}$ Significant at 0.05 level, ${ }^{*}$ Significant at 0.10 level. CPLP_R1 ${ }^{*}$, CPLP_R2 ${ }^{*}$, CPLP_R3 ${ }^{*}$, (CPLP_R1+KSP_R1) ${ }^{*}$, (CPLP_R2+KSP_R2) ${ }^{*}$ and (CPLP_R3+KSP_R3) ${ }^{*}$ are the maximum values of CPLP_R1, CPLP_R2, CPLP_R3, (CPLP_R1+KSP_R1), (CPLP_R2+KSP_R2) and (CPLP_R3+KSP_R3).

Table 5. $\mathrm{n}=25$; $\mathrm{df}=24$; $\mathrm{t}$ table of critical values.

\begin{tabular}{ccc}
$\alpha=0.005$ & $\alpha=0.05$ & $\alpha=0.1$ \\
$99 \%$ & $90 \%$ & $80 \%$ \\
2.797 & 1.711 & 1.318 \\
\hline
\end{tabular}

Table 6. Results, theorems, conjectures.

\begin{tabular}{cc}
\hline Table No. & Supported Empirical Investigation \\
\hline 1. & Result 1, 2, 3, 4 \\
& Theorem 1 \\
2. & Result 5,6 \\
& Theorem 2, 3 \\
& Conjecture 1, 2 \\
& Result 7, 8 \\
& Theorem 4, 5 \\
4. & Conjecture 3, 4 \\
& Result 9, 10 \\
Miscellaneous & Theorem 6, 7 \\
& Conjecture 5, 6 \\
\end{tabular}


investigation. Theoretical results say that R1 $<$ R2 $<$ R3 in general; but in some cases this was not observed (as found by Verma and Sharma [5]).

\section{References}

[1] Verma, P. and Sharma, R.R.K. (2008) Two Stage Capacitated Warehouse Location Problem: Conceptual Scheme of the Vertical Decomposition Approach. Proceedings of the 4th International Conference on Logistics and Supply Chain Management 2008, PSG College of Technology Coimbatore and Central Michigan University, USA.

[2] Geoffrion, A.M. and Graves, G.W. (1974) Multicommodity Distribution System Design by Benders Decomposition. Management Science, 20, 822-844. http://dx.doi.org/10.1287/mnsc.20.5.822

[3] Sharma, R.R.K. (1991) Modeling a Fertilizer Distribution System. European Journal of Operational Research, 51, 2434. http://www.sciencedirect.com/science/article/pii/037722179190142I http://dx.doi.org/10.1016/0377-2217(91)90142-I

[4] Sharma, R.R.K. and Berry, V. (2007) Developing New Formulations and Relaxations of Single Stage Capacitated Warehouse Location Problem (SSCWLP): Empirical Investigation for Assessing Relative Strengths and Computational Effort. European Journal of Operational Research, 177, 803-812. http://www.sciencedirect.com/science/article/pii/S037722170600018X http://dx.doi.org/10.1016/j.ejor.2005.11.028

[5] Verma, P. and Sharma, R.R.K. (2011) Vertical Decomposition Approach to Solve Single Stage Capacitated Warehouse Location Problem (SSCWLP). American Journal of Operations Research, 1, 100-117. http://www.scirp.org/journal/PaperInformation.aspx?paperID=7698\#.U01DvHbedk4 http://dx.doi.org/10.4236/ajor.2011.13013

[6] Sahin, G. and Sural, H. (2007) A Review of Hierarchical Facility Location Models. Computers and Operations Research, 34, 2310-2331. http://www.sciencedirect.com/science/article/pii/S0305054805002959 http://dx.doi.org/10.1016/j.cor.2005.09.005

[7] ReVelle, C.S. and Eiselt, H.A. (2005) Location Analysis: A Synthesis and Survey. European Journal of Operational Research, 165, 1-19. http://www.sciencedirect.com/science/article/pii/S0377221704002139

[8] ReVelle, C.S., Eiselt, H.A. and Daskin, M.S. (2008) A Bibliography for Some Fundamental Problem Categories in Discrete Location Science. European Journal of Operational Research, 184, 817-848. http://www.sciencedirect.com/science/article/pii/S037722170700080X

[9] Brandeau, M.L. and Chiu, S.S. (1989) An Overview of Representative Problems in Location Research. Management Science, 35, 645-674. http://pubsonline.informs.org/doi/abs/10.1287/mnsc.35.6.645 http://dx.doi.org/10.1287/mnsc.35.6.645

[10] Sharma, R.R.K. (1996) Chapter 5. Foodgrains Distribution in the Indian Context: An Operational Study. In: Tripathy, A. and Rosenhead, J., Eds., Operations Research for Development, New Age International Publishers, Ahmedabad, New Delhi, 212-227.

[11] Sharma, R.R.K. and Namdeo, S. (2005) Two Stage Capacitated Warehouse Location Problem: Developing New Strong Constraints. Proceedings of 5th International Conference on Operational Research for Development: ICORD V, Jamshedpur, Jamshedpur, 19-21 December 2005, 330-333.

[12] Sharma, R.R.K. and Sharma, K.D. (2000) A New Dual Based Procedure for the Transportation Problem. European Journal of Operational Research, 122, 611-624. http://www.sciencedirect.com/science/article/pii/S0377221799000818 http://dx.doi.org/10.1016/S0377-2217(99)00081-8

[13] Verma, P. and Sharma, R.R.K. ( 2007) Vertical Decomposition Approach to Solve Single Stage Capacitated Warehouse Location Problems. Proceedings of the IEEE International Conference on Industrial Engineering and Engineering Management (IEEM), Singapore, 2-4 December 2007, 907-911. http://ieeexplore.ieee.org/xpls/abs_all.jsp?arnumber=4419323\&tag=1

[14] Cornuejols, G., Sridharan, R. and Thizy, J.M. (1991) A Comparison of Heuristics and Relaxations for the Capacitated Plant Location Problem. European Journal of Operational Research, 50, 280-297. http://www.sciencedirect.com/science/article/pii/037722179190261S http://dx.doi.org/10.1016/0377-2217(91)90261-S

[15] Christofides, N. and Beasley, J.E. (1983) Extensions to a Lagrangean Relaxation Approach for the Capacitated Warehouse Location Problem. European Journal of Operational Research, 12, 19-28.

http://www.sciencedirect.com/science/article/pii/0377221783901790 http://dx.doi.org/10.1016/0377-2217(83)90179-0

[16] Nauss, R.M. (1978) An Improved Algorithm for Capacitated Plant Location Problem. Journal of Operational Research Society, 29, 1195-1201. http://www.palgrave-journals.com/jors/journal/v29/n12/abs/jors1978263a.html http://dx.doi.org/10.1057/jors.1978.263 